\title{
Émigration, colonisation et idéologie libérale en France (1840-1870)
}

Emigration, Colonization and Liberal Ideology in France (1840-1870)

Emigración, colonización e ideología liberal en Francia (1840-1870)

\section{Yves Charbit}

\section{(2) OpenEdition Journals}

\section{Édition électronique}

URL : https://journals.openedition.org/remi/7441

DOI : $10.4000 /$ remi.7441

ISSN : $1777-5418$

Éditeur

Université de Poitiers

\section{Édition imprimée}

Date de publication : 1 décembre 2015

Pagination : 209-230

ISBN : 979-10-90426-26-9

ISSN : 0765-0752

\section{Référence électronique}

Yves Charbit, «Émigration, colonisation et idéologie libérale en France (1840-1870) », Revue européenne des migrations internationales [En ligne], vol. 31 - n³ et 4 | 2015, mis en ligne le 01 décembre 2018, consulté le 14 avril 2022. URL : http://journals.openedition.org/remi/7441 ; DOI : https://doi.org/10.4000/remi.7441 


\section{Émigration, colonisation et idéologie libérale en France (1840-1870)}

\section{Yves Charbit ${ }^{1}$}

Cet article montre qu'au milieu du XIXe siècle l'évolution des idées sur l'émigration ne peut s'expliquer par les seuls changements démographiques, car elles ont été à la fois étroitement liées aux enjeux coloniaux et engluées dans l'idéologie. Une ferme contextualisation s'impose donc et ceci est particulièrement vrai des économistes libre-échangistes, en leur temps les meilleurs spécialistes de "la question de la population ", selon l'expression alors consacrée et bien plus juste que celle de " problème démographique ". Dans leurs écrits, empirie, théorie et idéologie sont indissociables et c'est cet enchevêtrement qui explique leurs spectaculaires volte-face, tant théoriques que doctrinales, sur l'émigration et la colonisation. Cas d'école presque pur de la pression exercée par l'idéologie sur la production des idées sur la population, ces revirements, telle une onde de choc, conduisirent à partir des années 1860 à une remise en cause radicale de la sacro-sainte allégeance à Thomas Robert Malthus.

Qui étaient les économistes libre-échangistes et de quel corpus disposonsnous ? Parmi le grand nombre de publicistes qui s'intéressèrent à la population française, ils méritent une attention particulière (Spengler, 1936 ; Charbit, 1981 et 2001 ; Breton et Lutfalla, 1991). À I'instar des libre-échangistes anglais, ils s'organisèrent à partir de 1841 en un groupe d'opinion et de pression pour obtenir l'abolition de la législation protectionniste qui n'avait cessé d'être renforcée depuis le XVIle siècle (Charbit, 2014). Très actifs, ils propagent leurs idées par leur presse : ils fondent en 1841 Le Journal des économistes, puis en 1846 I'hebdomadaire Le Libre-échange, pour soutenir la campagne anti-protectionniste ; en 1860 sont créés I'Économiste français et le Journal de la Société de statistique de Paris. Les économistes écrivent aussi régulièrement dans des quotidiens (Le Journal des débats) et des revues (La Revue des deux Mondes). Par ailleurs, ils tiennent pratiquement toutes les chaires, publiques et privées, d'économie politique ou de disciplines voisines. Outre leur solide implantation dans certaines sociétés savantes, telle l'Académie des Sciences morales et politiques, ils créent plusieurs Sociétés d'économie politique à Paris en 1842 puis en province (Marseille, Lyon, Bordeaux, Saint-Étienne et Douai) et une Société de statistique de Paris en 1860².

1 Professeur émérite de démographie, CEPED, Université Paris Descartes, 19 rue Jacob, 75006 Paris ; yves.charbit@ ceped.org

2 On utilisera les sigles suivants : Jde (Journal des économistes) ; Jssp (Journal de la Société de statistique de Paris) ; Rddm (Revue des deux Mondes) ; Asmp (Académie des sciences morales et politiques) ; Sep (Société d'économie politique). 
Ardents partisans du libre-échange, ils voient enfin leurs idées triompher à partir de 1860 grâce à I'appui politique de Napoléon III, avec la signature de plusieurs traités de commerce, en particulier avec l'Angleterre.

La période $1840-1870^{3}$ fut donc en quelque sorte celle de l'apogée du groupe. Mais les économistes méritent d'être étudiés pour deux autres raisons. Comme le critère rigide de l'appartenance au groupe était l'adhésion à la doctrine du libre-échange - on a pu parler de la "secte des économistes " -, il était possible à chacun d'avoir des positions beaucoup plus souples sur la question de la population sans risquer l'excommunication. D'où, pour nous, la précieuse possibilité de suivre les constants réajustements de leur doctrine de population aux évolutions tant structurelles que conjoncturelles. En témoignent les thèmes retenus, entre 1842 et 1870, pour les débats qui suivaient les dîners mensuels de la Société d'économie politique de Paris et qui furent régulièrement reproduits dans le Journal des économistes. Ces comptes rendus constituent un excellent indicateur de l'évolution des idées. C'est ainsi qu'après 1860, de plus en plus de débats furent consacrés à l'émigration et à la colonisation. Deuxième raison, contrairement au siècle précédent, leurs adversaires traditionnels, les protectionnistes d'inspiration néo-mercantiliste, sont avant tout des industriels et aucune figure de premier plan n'a élaboré une doctrine sociale et encore moins pris part aux débats sur la question de la population. Quant aux catholiques sociaux, bien étudiés par Duroselle (1951), ils n'abordent celle-ci qu'incidemment et le plus souvent à propos de la charité ou des enfants abandonnés et rarement de l'émigration et de la colonisation. Enfin, Proudhon mis à part, les socialistes utopiques, qui sont viscéralement anti-malthusiens, sont réduits au silence sous le Second Empire par la réaction policière.

Après un bref préambule d'ordre épistémologique, I'utilisation des arguments malthusiens est analysée, puis la relation entre libre échange et pacifisme et l'évolution vers l'impérialisme colonial. Des contradictions théoriques et doctrinales surgirent à partir de 1860 . Nous concluons par un bilan sur cette période privilégiée pour qui étudie les relations entre émigration et colonisation.

\section{Penser l'émigration et la population sans la démographie}

Les migrations sont aujourd'hui un champ en soi, où prédominent la sociologie, l'histoire, la géographie, l'économie, la science politique et plus récemment l'écologie avec la question des migrants climatiques, étant entendu que les recherches sont mono- ou interdisciplinaires. Puisque ces disciplines n'étaient pas toutes constituées dans le passé, le chercheur qui analyse aujourd'hui la genèse des théories et des doctrines se heurte très vite à une question : d'où jaillissaient les idées sur la mobilité, tant interne qu'internationale ? Nous avons montré ailleurs qu'elles s'inscrivaient, tout comme pour la fécondité et la mortalité, dans trois champs disciplinaires bien plus vastes que la démographie proprement dite et d'ailleurs antérieurs à elle, la philosophie

3 Soit, la Monarchie de juillet (1830-1848), l'éphémère Seconde République (1848-1851) et le Second Empire (1851-1870). 
politique, la philosophie morale, l'économie politique (Charbit, 2010)4

La philosophie politique est indispensable à la compréhension des idées sur la population et plus particulièrement des migrations, même si le lien n'est pas direct. La population et la migration surgissent dans la philosophie politique de la Grèce classique, chez Platon lorsqu'il s'interroge sur la construction de la Cité (Charbit, 2001) et chez Aristote (Kreager, 2008) quand il définit l'organisation de I'État. Il est remarquable que chez Platon la migration est l'objet de longs développements dans Lois, les Crétois demandant conseil à un Athénien sur la meilleure façon de fonder une colonie ${ }^{5}$. L'autre grande question de la philosophie politique est celle du pouvoir, qui se définit à travers les siècles comme une relation de domination entre son détenteur et ceux sur qui il s'exerce. La population est soumise au Prince qui va en tirer trois utilités : elle doit lui payer des impôts, se battre dans ses armées, lui fournir de la main-d'œuvre. L'immigration va ainsi lui fournir des artisans qualifiés et leurs secrets de fabrication et l'émigration lui permettre d'étendre le territoire sur lequel il règne, argument particulièrement important dans le contexte de l'émergence des grands États européens et de leurs rivalités exacerbées par le partage du Nouveau Monde après les grandes découvertes de la fin du XVe siècle. Ce scénario se rejouera au XIXe siècle avec la conquête et la colonisation de l'Afrique. La philosophie morale n'est pas moins essentielle. Lorsqu'au XVIIle siècle émerge la notion d'individu, comprendre les mobiles de son comportement va conduire à proposer l'idée de rationalité, d'une rationalité séculière profondément différente de la morale chrétienne définie par rapport à Dieu et qui privilégiait la contemplation. L'activité économique devient légitime et l'individu peut se donner pour objectif de maximiser son intérêt. On le sait, Weber alla jusqu'à parler de l'éthique protestante du capitalisme. Enfin, l'économie politique apporta en complément de la philosophie morale un riche panier de concepts et d'outils, dont le marché, la valeur, I'emploi, etc. Cet approfondissement de la morale utilitariste supposait l'identification des paramètres démographiques. Ainsi, se construisit, en particulier avec Malthus, un homo demographicus complémentaire de I'homo ceconomicus d'Adam Smith, car I'utilitarisme économique s'appliquait non seulement au comportement économique de l'individu, mais aussi à son comportement démographique. Cela est vrai de la fécondité - Jean-Baptiste Say recommandait aux pauvres de faire moins d'enfants et plus d'épargnes -, tandis que l'émigration se lit aisément comme un avatar de I'utilitarisme, l'individu cherchant à maximiser son intérêt en émigrant pour faire fortune. De même, l'intérêt bien compris des États était de faciliter la libre circulation des produits et des hommes. Mais au début du XIXe siècle et jusque dans les années 1860, les connaissances sur les migrations internationales étaient encore balbutiantes et les rares données disponibles ne furent pas exploitées de manière systématique et encore moins théorisées. Depuis I'Essai sur le principe de population publié par Malthus en 1798, I'émigration n'était qu'une des composantes de la dynamique des populations, à côté de la natalité et de la mortalité, la seule question étant de savoir si elle pouvait ou non contribuer à freiner la croissance de la population. Et comme chez Malthus, considérations doctrinales et arguments théoriques se renforçaient.

4 Rappelons que la naissance de la démographie est en général attribuée à John Gaunt, avec l'invention des tables de mortalité, le terme de démographie apparaissant chez Adolphe Guillard en 1856.

5 Platon, Lois, 708a-d ; 735a-736c ; 777c-d ; 704d-705a ; 950a-952 ; 952d-953e ; 950b. 


\section{La problématique malthusienne de l'allocation des ressources}

Malthus avait eu un certain nombre de prédécesseurs, mais il fut le premier à modéliser les interactions entre variables démographiques et rationalité économique ; mieux, à montrer que les variables démographiques faisaient système, en tout cas au minimum la fécondité, la nuptialité, la mobilité, tandis que la mortalité apparaissait en son temps comme devant être subie, sans réelle possibilité d'échapper à la maladie et à son issue fatale. La peste, parmi d'autres épidémies, est archétypale : jusqu'à Hiersin, elle fut un fléau récurrent en face duquel I'homme était impuissant... sauf à fuir, pour éviter la contagion, fuite qu'il est tout à fait légitime d'analyser comme une forme de migration, au même titre que les déplacements de population induits aujourd'hui par les famines ou les catastrophes climatiques.

Au cœur de la pensée de Malthus se trouve la comparaison entre la croissance géométrique de la population (doublement tous les vingt-cinq ans soit un taux annuel moyen égal à $2,8 \%$ ) et la croissance arithmétique, beaucoup plus lente, des subsistances. Fondamentalement, la pensée de Malthus est dynamique. Certes, il y a forcément équilibre, mais, dit-il, la population a constamment tendance à dépasser les subsistances en raison de ce qu'il appelle le principe de population et que nous pouvons raisonnablement assimiler à la force de l'instinct sexuel : dès que des subsistances sont disponibles, la population augmente. Dans les espèces animales, qui ne connaissent pas le contrôle de la raison sur l'instinct, mais aussi au sein du prolétariat, qui, selon Malthus, se reproduit de manière inconsidérée, ce déséquilibre ne peut à ses yeux se résoudre que par la sanction de la mortalité. Dans les classes moyennes au contraire, la pratique de la " contrainte prudente ", entendons la contraception, exerce un frein préventif en limitant la fécondité. La théorisation de l'émigration s'inscrit parfaitement dans cette conceptualisation théorique de la croissance démographique. L'émigration vers de nouveaux territoires équivaut à accroître les subsistances et permet un nouvel accroissement démographique. Malthus cite expressément les nouveaux États d'Amérique du Nord comme I'exemple type d'une croissance démographique non limitée par les subsistances. Partout ailleurs, écrit-il, cette croissance a été limitée. Pour réfuter l'argument qui lui fut opposé par les radicaux anglais tels William Godwin (Political Justice, 1793 et On Population, 1820), pour qui précisément les possibilités de mise en valeur de nouvelles terres desserraient l'étau du principe de population, Malthus répliqua que l'émigration ne pouvait être une solution, car tôt ou tard la planète serait intégralement peuplée, argumentaire repris par Paul Ehrlich (1968) et le Club de Rome (Rapport Meadows de 1972), et qui est sous-jacent aux débats actuels sur le développement durable.

Notons que la conceptualisation de l'émigration par Malthus en 1798 est purement démographique, car il ne fait nullement intervenir les analyses économiques en termes de demande de travail qui seront centrales dans l'évolution de sa pensée, qu'il s'agisse des éditions ultérieures de l'Essai sur le principe de population (la seconde parut en 1803) ou des Principes de l'économie politique (1820). Malthus fut introduit en France au tout début du XIXe siècle par Say et durant les décennies 1840 et 1850 ses idées triomphent. Mais les économistes ne se contentèrent pas de les répéter sans vraiment innover. Ils enrichirent la réfu- 
tation de l'émigration en s'appuyant sur la théorie de la répartition du capital. En effet selon la théorie classique des marchés, l'équilibre se produit au point où I'offre est égale à la demande. Dans le cas de ce produit particulier qu'est le travail, le salaire définit ce point d'équilibre entre l'offre de main-d'œuvre par les salariés et la demande de main-d'œuvre par les entreprises. Conformément à la théorie de Smith, tant que ce point d'équilibre n'est pas atteint, les salariés vont augmenter leur fécondité pour répondre aux possibilités d'emploi. En un mot, c'est la demande qui gouverne l'offre, ou encore il n'y a pas de croissance démographique sans croissance économique ${ }^{6}$. Tel est le soubassement théorique de la notice "Colonisation " du Dictionnaire de l'économie politique, paru en 1853 (I : 393-401) où I'auteur, Gustave de Molinari, s'opposait au financement par l'État de la colonisation :

«En subventionnant la colonisation, les gouvernements de l'Europe dépouillaient certaines branches du travail pour en favoriser d'autres, qui étaient en réalité moins productives dans les conditions où ils les plaçaient; ils provoquaient une distribution moins profitable des fonds productifs, partant une diminution de la richesse ".

On observera que Molinari passait totalement sous silence le fait que les colonies permettaient d'accroître la production. Celles-ci s'analysent en effet en un élargissement des marchés, à la fois source d'approvisionnement en matières premières pour la métropole et débouché pour ses produits manufacturés. Ici encore, il faut revenir à la théorie économique dominante à l'époque, car elle empêchait de comprendre le fonctionnement économique des colonies. Selon la " loi des marchés " de Say, les produits finissent toujours par s'échanger contre des produits et la monnaie n'est qu'un voile ; il en résultait que la migration ne pouvait créer de la richesse et la vraie question était bien d'optimiser l'affectation du capital pour maximiser le taux de profit.

Mais précisément, poser la question de cette optimisation, c'était raviver de brûlants débats doctrinaux fortement teintés d'idéologie : dans cette France dominée par les intérêts protectionnistes emmenés par un front commun de maîtres de forges, d'industriels et de propriétaires fonciers, les libre-échangistes s'étaient toujours posés en défenseurs des consommateurs et donc du sacrosaint intérêt général. Quand avait éclaté la révolution de février 1848, ils avaient accusés les protectionnistes d'entretenir la misère : les tarifs protecteurs créaient une " cherté artificielle " des produits de consommation courante et en particulier des subsistances, question éminemment sensible. Les Français devraient-ils, comme les Irlandais, connaître la famine et la misère et abandonner leur pays plutôt que mourir littéralement de faim ?

\footnotetext{
6 Dans le chapitre consacré aux salaires du travail, écrit en 1776 dans la Richesse des Nations : "Si cette demande augmente continuellement, la rémunération du travail doit nécessairement encourager le mariage et la multiplication des travailleurs de telle manière qu'elle leur permette de répondre à cette demande en accroissement constant par une population en augmentation constante. [...] La demande des hommes, comme celle de n'importe quel autre bien, en régule nécessairement la production. Elle l'accélère quand elle croît trop lentement, et elle l'arrête quand elle progresse trop rapidement " (Smith, 1970 : 183 - Livre 1, chap. 8).
} 


\section{Libre échange et pacifisme}

Mais pourquoi les colonies suscitaient-elles une réelle hostilité et non pas un simple manque d'intérêt chez les économistes ? Le contexte politique et les considérations idéologiques sont ici décisifs. Jusqu'à la suppression du Pacte colonial (loi du 31 juillet 1861), I'existence de colonies impliquait tout un système monopolistique. L'identification de la colonisation au Pacte colonial était si forte qu'en avril 1864 encore un débat eut lieu à la Société d'économie politique sur le thème suivant : "Les économistes ont-ils confondu de manière incorrecte les colonies, le système colonial et la colonisation "(Annales de la Sep, 5 : 710). En clair, les colonies étaient acceptables si elles étaient organisées selon les principes du libre-échange. L'importance du changement dans la politique commerciale du Second Empire et en particulier du traité de libre-échange signé avec I'Angleterre en janvier 1860 ne saurait en effet être sous-estimée. II devenait possible de justifier les migrations et les colonies à un niveau théorique en relation avec l'établissement du libre-échange et le monde allait devenir un vaste et unique marché des produits et des hommes, comme en témoignent ces lignes écrites en 1863 :

"Les clôtures qui séparent les différents pays tant pour les hommes que pour les choses, s'abaissent et tendent à disparaître, à la fois par le progrès inouï des voies de communication et par la suppression graduelle des entraves du régime prohibitif. Des millions d'Européens, par exemple, se sont répandus depuis cinquante ans dans les autres parties du globe pour y offrir leurs services : un plus grand nombre d'autres y ont trouvé un débouché croissant pour leurs produits, ce qui a agrandi d'autant le marché de leurs populations en Europe même. Des millions d'Asiatiques et d'Africains ont, de même été transportés, soit de gré, soit de force, dans d'autres régions du globe devenues ainsi des annexes au marché primitif de leur population " (Molinari, 1864, I : 406).

Le cas de Frédéric Passy révèle comment la convergence de facteurs historiques, précis et parfaitement identifiables, et de considérations idéologiques rend compte de l'évolution de l'anticolonialisme au colonialisme. En 1855, il était hostile aux colonies en raison de leurs conséquences démographiques sur le pays d'origine. Contre Watteville qui soutenait que les émigrants devraient être qualifiés et industrieux, il affirma :

"De pareils hommes sont une richesse pour un pays et on ne comprend pas quel avantage il peut y avoir à les en faire sortir. Quant aux pauvres ils coûtent plus chers à expatrier qu'à nourrir dans leur pays " (Passy, $1857: 171)^{7}$.

Le premier argument renvoie à la critique libre-échangiste du Pacte colonial.

En effet, " les colonies qui ne sont rien si elles ne sont un débouché commercial, sont le plus illusoire de tous les débouchés et si un certain nombre d'individus peuvent y trouver leur compte, la nation est toujours en perte pour une somme plus forte "

(Passy, $1857:$ 157).

7 Les Mélanges d'économie politique sont un recueil d'articles parus en juin, août et septembre 1855 dans le Jde. 
Dans cette perspective, mieux valait, certes, conserver la main-d'œuvre qualifiée et exporter seulement les pauvres. Le deuxième argument est intéressant, car il fait écho aux thèses malthusiennes : l'émigration ne peut être une solution aux problèmes sociaux. Passy fit allusion aux " désastreuses tentatives de colonisation " de l'Algérie en 1848 (Passy, 1857 : 171). Effectivement, sous la Seconde République, un crédit de 50 millions de francs avait été ouvert au budget pour le transport et l'installation en Algérie de nécessiteux (décret du 23 septembre 1848). Cette tentative échoua à la fin de la Seconde République et la moitié des 21000 émigrants étaient revenus en France (Julien, 1966 : 362-377).

En 1867, I'opinion de Passy avait considérablement évolué, bien qu'il continuât à proclamer son hostilité à la colonisation : dans sa Conférence sur la paix et la guerre, il affirma qu'aucun pays n'avait réellement retiré des bénéfices de ses colonies, ni l'Espagne avec ses possessions d'outre-Atlantique, ni la France (Passy, 1867 : 13). Cependant, il admit la nécessité de protéger les marchés. C'était là un changement doctrinal majeur et derrière les arguments humanitaires le point de vue colonialiste ne fait aucun doute :

« Le canon n'est pas le meilleur moyen pour ouvrir les marchés. J'admets certes que I'on veuille faire triompher la civilisation de la barbarie ; mais ce n'est pas par la terreur c'est par les lumières, c'est par les capitaux, par exemple, qu'il peut s'obtenir. De bons traitements de bons exemples des procédés de culture, de sciences, de médecine, des enseignements moraux, du respect, de la dignité, de la probité en un mot, voilà, à mon avis, comment on ouvre des débouchés et surtout comment on les conserve "

(Passy, $1867:$ 12).

L'évolution de Passy s'explique aisément. Ce pacifiste convaincu, qui fut l'un des fondateurs de la Ligue internationale pour la paix, dont il devint le Secrétaire général et qui partagera en 1901 le prix Nobel de la paix avec Henri Dunant, s'était senti contraint de prendre position sur la question de la décadence de la France.

" On vous parle de conquête il est beau de s'agrandir vous dit-on, c'est la preuve de la vitalité d'une nation; et toute race qui n'est pas atteinte de décrépitude est naturellement expansive " (Passy, $1867: 12$ ).

Que s'était-il passé ? La guerre avait éclaté entre la Prusse et l'Autriche autour des duchés du Schleswig-Holstein et les troupes prussiennes avaient écrasé celles de l'Empire austro-hongrois à Sadowa le 3 juillet 1866. Selon I'expression consacrée, la victoire de la Prusse fit l'effet d'un " coup de tonnerre dans le ciel serein du Second Empire ", car aux portes de la France se révélait brutalement une puissance militaire, scientifique et industrielle qui avait en outre pour stratégie d'établir son hégémonie sur l'ensemble des principautés et royaumes allemands. Immédiatement après Sadowa, fut créée une Confédération de l'Allemagne du Nord dominée par la Prusse (Armengaud, 1962 : 2).

Plaider en faveur d'une colonisation " non-violente " était donc, après Sadowa, un habile moyen de concilier libre-échange et grandeur de la France. En 1869, à la veille même de la guerre de 1870 contre la Prusse, Passy est encore plus clairement favorable à la colonisation et il en oppose de manière lyrique les bienfaits aux " maux de la guerre " : 
"Ce n'est pas là le véritable patriotisme, la véritable ambition, ce n'est pas ainsi qu'on occupe une plus large place sur le globe. S'ouvrir des chemins à travers les déserts et les forêts vierges qui barrent devant nous les trois quarts du globe, fertiliser les pays si nombreux encore dans lesquels la race humaine n'a pas mis les pieds, appeler à l'éclosion de la grande vie intellectuelle et morale ces frères que nous nommons barbares, ce sont là des victoires qu'il nous reste à remporter, et que nous remporterons espérons-le car ces idées gagnent le du terrain " (Passy, 1869 : 63-64).

En résumé, I'hostilité totale qui prévalait à l'encontre de la colonisation jusqu'en 1860 s'appuyait sur les thèses malthusiennes et était centrée sur le problème de la misère. Vers la fin du Second Empire, le renouveau du thème colonial est au contraire directement lié au contexte international.

\section{Vers l'impérialisme colonial}

L'instauration du libre-échange en 1860 fut directement à l'origine de ce retournement doctrinal, mais le renouveau du colonialisme s'inscrit dans un contexte historique et idéologique plus vaste. À la différence de l'Angleterre, la France possédait peu de colonies vers 1848. À part la Guadeloupe, la Martinique, Saint-Pierre-et-Miquelon, quelques postes sur la côte de la Guyane, l'île de la Réunion, cinq établissements en Inde, quelques-uns sur les côtes de l'Afrique (Sénégal, Guinée, Gabon), des points de relâche aux Comores à Tahiti et dans les îles Marquises, la seule possession de quelque ampleur territoriale était I'Algérie surtout après la reddition d'Abdelkader en 1847. Bien qu'incohérente, la politique coloniale du Second Empire fut au total beaucoup plus vigoureuse que celle des régimes précédents. Si l'on met à part la déportation de républicains en Algérie après le coup d'État du 2 décembre, la première décision importante fut la création en 1858 d'un ministère de l'Algérie et des colonies, dirigé par le prince Napoléon, puis par Chasseloup-Laubat. Au Sénégal, la colonisation fut pratiquement l'œuvre d'un seul homme, Faidherbe, qui réussit en quelques années (il ne fut gouverneur qu'en 1854-1861 et 1863-1865) à asseoir l'influence française avec un minimum de soutien armé. Entre 1850 et 1865, le commerce avec le Sénégal tripla et celui-ci devint une colonie de production, consacrée à la culture des arachides. En Extrême-Orient, la conquête du Cambodge et de la Cochinchine fut réalisée entre 1859 et 1867. Madagascar fut annexée en 1868. Enfin, la France s'implanta au Moyen-Orient.

La politique coloniale suscita des réactions contradictoires dans l'opinion publique et plus précisément au sein du groupe des économistes. D’un côté, les réactions furent négatives, soit en raison d'une hostilité purement politique au Second Empire, la question coloniale servant alors de prétexte à l'expression d'une opposition, soit parce que la colonisation était jugée peu souhaitable économiquement, car il était admis que l'Algérie coûtait bien plus cher à la France qu'elle ne rapportait, soit enfin pour des motifs d'ordre idéologique comme au Siècle des Lumières, les colonies et l'esclavage étant la négation des droits de l'Homme. En sens inverse, I'expansion coloniale du Second Empire a pu correspondre à une certaine idée de la grandeur de la France, la politique coloniale de Napoléon III apparaissant non comme une fin en soi, mais comme un des éléments de la politique intérieure et extérieure, les succès obtenus contribuant à renforcer le régime. Dans cette perspective, un facteur essentiel 
fut la rivalité avec la Grande-Bretagne, car la grandeur coloniale de la France se mesurait nécessairement à l'aune de l'Empire britannique.

\section{Faire pièce à l'Angleterre}

Bien que les chiffres soient peu sûrs, l'écart entre les deux puissances était incontestable. Les données dont disposaient les économistes étaient, par exemple, celles publiées par Émile de Laveleye en 1865 dans le Journal de la Société de statistiques de Paris (Tableau 1).

Tableau 1 : La population des deux Empires coloniaux en 1865

\begin{tabular}{|l|l|}
\hline France & $\mathbf{3 7 4 7 2} \mathbf{7 6 2}$ \\
\hline Afrique (Algérie) & 2999124 \\
\hline Afrique (Sénégal, Réunion, Ste-Marie-Margotte) & 255463 \\
\hline Asie (Inde et Basse Cochinchine) & 2221507 \\
\hline Amérique (Martinique, Guadeloupe) & 300162 \\
\hline Pacifique (Marquises, etc.) & 84460 \\
\hline Total & $\mathbf{4 3 3 3 3 4 7 8}$ \\
\hline \multicolumn{2}{|l|}{} \\
\hline Grande-Bretagne & $\mathbf{2 9 3 0 7 2 0 0}$ \\
\hline Europe & 158854 \\
\hline Asie & 137673494 \\
\hline Afrique & 914360 \\
\hline Amérique & 4422261 \\
\hline Australie & 1358831 \\
\hline Total & $\mathbf{1 7 3 8 3 5 0 0 0}$ \\
\hline \multicolumn{2}{|l}{ Source : Jssp, 1865: 159. } \\
\hline
\end{tabular}

La comparaison avec l'Angleterre ne se limitait pas à de simples considérations d'ordre politique ou démographique. Le cas de cette puissance rivale était exemplaire d'un autre point de vue : les observateurs perspicaces ne pouvaient ignorer l'importance des colonies pour l'économie du pays. En Angleterre d'ailleurs, le courant en faveur des colonies a été sans doute beaucoup plus important que les énergiques prises de position anticoloniale de Jérémie Bentham, James Mill, Richard Cobden ou J. R. McCulloch ne le laissent penser (Shaw, $1970: 23,19$ et 25). En réalité, on peut parler avec John Gallagher et Ronald Robinson de "l'impérialisme du libre-échange " : I'empire informel du commerce et de l'investissement a recueilli bien plus d'adhésions que la politique coloniale proprement dite (1970:34).

II convient ici de rappeler quelques faits. La répartition géographique des échanges entre le Royaume-Uni et le reste du monde entre 1830 et 1880 montre la forte croissance des marchés coloniaux malgré l'établissement du libre-échange en Europe en 1860 (Tableau 2). Le poids de I'Asie s'explique par I'importance du marché indien et par la destruction de l'industrie textile locale. L'accroissement brutal de la part de l'Océanie entre 1830 et 1860 résulte des découvertes en Australie des gisements aurifères, qui induisit une demande pour le marché intérieur de ce pays (Bairoch, 1976 : 193). Le cas de l'industrie cotonnière, la plus développée et la plus représentative de la révolution industrielle, est encore 
plus frappant : c'est le triomphe des marchés extérieurs sur le marché domestique (Hobsbawm, 1977 : 50). En 1815, I'Angleterre exportait quatre mètres de coton quand elle en consommait trois. En 1851, elle en exportait treize contre huit. En 1820, l'Europe importait 128 millions de mètres ; I'Amérique (États-Unis exceptés), I'Afrique et I'Asie, 80 millions. En 1840, les chiffres étaient respectivement de 100 et 129 millions. Le marché indien entre ces deux dates passa de 11 à 145 millions de mètres. En valeur, les produits cotonniers représentaient près de $50 \%$ de toutes les exportations britanniques dans les années 1840 (Hobsbawm, 1977 : 50-54). Dans ces conditions, il n'est pas surprenant que les analyses de la colonisation aient fait, de manière explicite ou implicite, référence à l'Angleterre. À propos des résultats du recensement de 1866, le statisticien Maurice Block insista par exemple sur la nécessité, lorsque I'on comparait la France à I'Angleterre ou l'Allemagne, de prendre en compte " les centaines de mille ou les millions qui ont fait fortune ailleurs".

Tableau 2 : Répartition géographique des échanges extérieurs du Royaume-Uni

\begin{tabular}{|l|l|l|l|l|l|l|l|}
\hline & Europe & $\begin{array}{c}\text { Amérique } \\
\text { du Nord }\end{array}$ & $\begin{array}{c}\text { Amérique } \\
\text { du Sud }\end{array}$ & Asie & Afrique & Océanie & Total \\
\hline \begin{tabular}{l}
$\mid$ Exportations \\
\hline 1830
\end{tabular}$\quad 46,7$ & 25,5 & 11,5 & 12,8 & 2,5 & 1,0 & 100 \\
\hline 1860 & 34,3 & 16,6 & 12,0 & 25,7 & 3,2 & 8,2 & 100 \\
\hline 1880 & 35,6 & 15,9 & 10,2 & 25,4 & 4,3 & 8,4 & 100 \\
\hline \multicolumn{7}{|l|}{} \\
\hline 1860 & 31,0 & 26,7 & 10,1 & 23,2 & 4,5 & 4,5 & 100 \\
\hline 1880 & 41,4 & 30,9 & 6,1 & 12,0 & 3,7 & 5,9 & 100 \\
\hline
\end{tabular}

Source : Bairoch, $1976: 88$.

En 1865, Laveleye concluait une comparaison chiffrée des différents pays européens et leurs colonies par cette constatation :

" L'Empire britannique commande d'une manière plus ou moins directe dans toutes les parties du monde et sur des territoires dont l'ensemble dépasse un quart environ de la surface du globe " (Jssp, $1865:$ 159-160).

Les économistes pouvaient lire dans le Journal la société de statistiques de Paris de très nombreuses informations sur les migrations anglaises, les progrès des colonies, les forces militaires de l'Empire britannique ${ }^{8}$. Mais c'est Charles Lavollée, le chef du Bureau de l'émigration au ministère de l'Intérieur, qui analysa le mieux les relations entre industrialisation et colonisation, en faisant explicitement référence à I'Angleterre. Certes, les colonies n'étaient pas nécessaires au développement économique d'un pays, mais

" II n'en demeure pas moins certain que plusieurs nations d'Europe sont en grande partie redevables de leur prospérité, de leur influence politique, à la situation favorable des territoires qu'elles occupent au-delà des mers. Se figure-t-on ce que serait l'Angleterre si elle n'avait point de colonies ? [...] Le mouvement [...] industriel de la Grande-

8 Jssp, mai 1866 : 125-126 ; novembre $1866: 291-296$; septembre $1868: 226$; octobre $1868: 265-266$; janvier $1969: 28$; juin $1868: 149$; juillet $1869: 189$; août $1869: 218-219$; octobre 1869 : 264-265 ; novembre $1869: 278-280$. 
Bretagne et le commerce de la Hollande eussent été étouffés dans leur germe, s'ils n'avaient pu s'étendre par-delà les étroites limites de l'Europe et trouver en Amérique et en Asie d'inépuisables ressources d'expansion $"^{9}$.

Lavollée rappela la double fonction des colonies. Elles fournissaient des matières premières à l'industrie de la puissance coloniale, tout en constituant un marché pour ses produits :

"Ainsi, considérée au point de vue économique, la colonisation ne cause aucun préjudice à la fortune d'un État, et l'on peut ajouter que pour les grandes nations européennes elle est devenue une nécessité de premier ordre. Elle est la conséquence de l'immense mouvement industriel qui [...] a décuplé le travail des manufactures. II faut à tout prix accroître les approvisionnements en matières premières et créer des débouchés pour les produits. Les colonies répondent à ce double besoin en activant sur tous les points du globe les progrès de la culture de la consommation et des échanges".

Et contrairement à la thèse anticolonialiste, l'immigration permettait une meilleure répartition des richesses nationales.

"Quoi qu'il en soit, si certains mauvais esprits conservent quelques doutes quant à l'influence favorable ou désavantageuse qu'exerce sur la prospérité d'un État l'émigration vers l'étranger, ce doute doit disparaître lorsque l'émigration a lieu d'une métropole à destination d'une colonie. Dans ce cas particulier, tout est profit pour la métropole. Il y a là pour elle, non point une diminution, mais un déplacement et une répartition meilleure de la population du capital ce qui doit amener une augmentation de richesse. L'émigrant qui s'établit dans une colonie ne cesse point d'appartenir au domaine national : directement ou indirectement, il continue à fournir sa quote-part au revenu de l'État; sur un autre sol et sous d'autres formes, il demeure contribuable en même temps que citoyen, et les fruits de son travail sur la terre lointaine viennent en accroissement des forces productives de la mère patrie. II n'est plus nécessaire de développer à cet égard une démonstration théorique ; le capital emporté par le colon retourne à la métropole ; il lui est rendu avec usure par le moyen des échanges qui ne tardent pas à s'établir ".

Ainsi, les considérations doctrinales de nature géopolitique et économique se justifiaient par la théorisation de la nouvelle allocation optimale des ressources et des facteurs de la production. L'argumentaire d'inspiration malthusienne était bel et bien devenu obsolète sous la pression des faits.

\section{L'Algérie, fleuron de l'Empire}

L'Algérie, en dépit des désillusions initiales, était porteuse de bien des espoirs. Sa proximité géographique, l'importance numérique des colons, l'ampleur des territoires concernés en faisaient la meilleure chance coloniale de la France. En 1869 par exemple, le Jssp publia des chiffres sur le "Mouvement commercial de l'Algérie en 1868 »; il avait aussi reproduit par deux fois, en 1863 et 1868, des

9 Rddm, février 1863 ; les citations sont tirées des pages 883, 887 et 889. De même, Alfred Legoyt, dans un article du Jssp consacré au commerce de l'Angleterre en 1868, rappelant le " commerce immense " que celle-ci entretenait avec ses colonies, reproduisit les chiffres sur la part des colonies dans l'ensemble des importations et des exportations (Jssp, novembre $1869: 278-286$ ). 
discours mettant en évidence les résultats obtenus ${ }^{10}$.

Rappelons qu'à peine créé en 1858, le ministère de I'Algérie et des colonies fut supprimé en 1860. Les gouverneurs militaires, Pélissier puis Mac-Mahon, établirent alors une véritable dictature, tandis que les "Bureaux arabes " mettaient en œuvre la politique dite du cantonnement. Celle-ci eut pour résultat d'entraver la colonisation et suscita une vive opposition de la part des colons. Le Sénatus-consulte du 22 avril 1863 déclara que les tribus devraient acquérir les terres, tandis que celui du 14 juillet 1865 tentait de réconcilier les intérêts des colons et ceux des indigènes. L'Algérie, écrivait Napoléon à Mac-Mahon à son retour d'Algérie en 1865, est à la fois " un royaume arabe, une colonie européenne et un camp français ". Hippolyte Passy (Jde, 1869, $15: 125,127$ et 132) Nicolas Villiaumé (1867, II : 77), ou encore Alfred Legoyt (Jssp : 1862 : 258), le chef du Bureau de la statistique tout au long du Second Empire, ne manquèrent pas de souligner l'absence de "libertés administratives et commerciales " et accusèrent le " despotisme militaire " d'être responsable de la lenteur des progrès de la colonisation, spécialement en Algérie ${ }^{11}$.

L'Algérie apparaissait certes comme un marché potentiel pour les produits français (Batbié, 1866, II : 77), mais elle pouvait aussi affranchir l'industrie cotonnière de sa dépendance à l'égard des États-Unis pour son approvisionnement. Aussi, n'est-il pas surprenant, lorsque la Guerre de Sécession créa une " famine de coton " que la Société industrielle de Mulhouse, animée par Jean Dollfus, un actif libre-échangiste, ait fait des efforts pour favoriser le développement de la culture du coton (Fohlen, $1956: 347-355^{12}$ ). Anselme Batbié définit bien I'enjeu lorsqu'il écrit à propos de l'Algérie :

"Si la culture prend en Afrique assez de développement pour faire une concurrence sérieuse à l'Amérique, notre colonisation aura beaucoup fait pour l'affranchissement de l'industrie européenne. Alors nous serons indemnisés par les résultats économiques des sacrifices que nous avons semés pour faire avancer la civilisation "

(Batbié, 1866, II : 316-317) ${ }^{13}$.

Pour que se concrétisent ces espérances encore fallait-il que les hommes ne fissent point défaut pour la mise en œuvre des richesses de l'Algérie. Dès 1852, Lavollée avait déploré le fait que l'Algérie ait servi uniquement à se débarrasser des opposants politiques et des indigents en surnombre. Une colonisation inspirée du système Wakefield permettrait au contraire de la faire prospérer. Ce système consistait à financer l'émigration par la vente de terres de la couronne aux colons. II fut appliqué en Nouvelle-Zélande et en Australie du Sud dans les années 1830 (Rddm, 1852 : 128). Le système Wakefield qui suscita des controverses en Angleterre ${ }^{14}$ fut prôné par Villiaumé (1867, II : 74-77) et, au nom du grand principe libéral d'abstention de l'État gendarme, critiqué par Bénard, Jules Duval, Joseph Juglar comme trop interventionniste (Jde, 1869, 15 : 125-131).

10 Jssp, septembre $1869: 228-230 ; 1863: 224-225$ et $1868: 136$.

11 Sur les excès commis par les militaires voir Brunschwig, $1949: 22-27$ et 38-40.

12 Fohlen souligne également le rôle du Jde, de la Rddm, de la Revue contemporaine.

13 Même opinion chez Legoyt, Jssp, juillet $1865: 201$.

14 Voir Winch, 1963 et Gosh, 1964. 
La question de la colonisation débouchait donc sur celle de l'émigration et des possibilités de survie des nouveaux immigrants. II est frappant de constater l'importance au cours des années 1860 du thème de " l'acclimatement " des Européens à d'autres continents et en particulier en Afrique, thème développé à l'intérieur comme à l'extérieur du groupe des économistes ${ }^{15}$. Legoyt (Jssp, janvier 1865 : 10-13; Jssp, avril 1865 : 104) et Adolphe Bertillon (Jssp, juillet 1864 : 178) montrèrent que les données démographiques justifiaient l'installation des Européens d'Algérie. Après trente-cinq ans d'excédent des décès, la mortalité semblait diminuer et un excédent de naissances avait été enfin enregistré.

\section{Contradictions théoriques et doctrinales}

Ce regain d'intérêt pour les colonies a eu une conséquence doctrinale fondamentale : la question de l'immigration n'a pratiquement jamais été traitée indépendamment de celle de la colonisation. Les migrations sont bien au contraire le moyen d'une fin, qui se justifie au niveau économique ou politique, mais qui dépasse en tout cas le cadre de la seule doctrine démographique.

\section{La remise en cause du malthusianisme}

II était pourtant concevable d'analyser l'émigration dans une toute autre perspective. On l'a dit, d'un point de vue malthusien, celle-ci était avant tout étudiée en tant que moyen de soulager le pays de départ d'une excessive pression démographique; et selon Malthus, ce moyen était totalement inefficace puisque le vide laissé par les émigrants serait immédiatement comblé par un regain de la natalité, en raison même du principe de population. Prôner la colonisation et l'émigration impliquait donc nécessairement de remettre en cause les thèses malthusiennes. Le cas de Joseph Garnier, le rédacteur en chef du Journal des économistes, est particulièrement révélateur. Ce malthusien orthodoxe avait utilisé l'argument traditionnel en 1846 au début de la crise qui allait emporter en février 1848 la Monarchie de Juillet :

"Au sujet des migrations et de la colonisation sur lesquels l'opinion publique semble tant compter aujourd'hui pour le soulagement du malaise je me bornerai à dire que Adam Smith, Malthus, Jean-Baptiste Say et M. Rossi ont réduit à leur véritable expression les services de second ordre qu'on peut en attendre. Elles sont coûteuses pour la société, tyranniques pour le pauvre qu'on exile, insuffisantes puisqu'elles ne retirent guère que quelque milliers d'hommes de certains pays où l'excès se mesure par millions "

(Jde, 1846, $15: 131$ ).

Mais dans les débats qui ont lieu à la Société d'économie politique en 1864 et 1869 , il est frappant de constater que sa contribution se limite à une critique du Pacte colonial alors qu'il resta silencieux sur l'émigration (Jde, 1869, 15 : 131). Or le Pacte colonial avait été supprimé en 1861, alors que l'émigration était bel et bien une question d'actualité ! On notera également dans la citation suivante la

15 À l'extérieur du groupe voir par exemple A. de Quatrefages, Rddm, avril 1861 : 635-731 ; Boudin (1852 et 1860) avait toujours nié la possibilité pour les races de $\mathrm{s}^{\prime}$ adapter à différents milieux. A. Martin et L. Foley avaient souligné la forte mortalité qui frappait à tous âges les immigrants (1851:341-342) ; J.-J. Weiss était plus nuancé (1857, 34 : 434-435). 
référence à la " conquête de l'Algérie " et la critique de la théorie mercantiliste de la colonisation, alors que Villiaumé venait de développer (Jde, 1869, 15 : 123-134) les avantages d'une émigration inspirée du système de Wakefield:

« M. Garnier rappelle que la théorie de l'enrichissement national par les possessions lointaines, par les colonies et par la colonisation, fille de la balance du commerce, a engendré les plus grands maux : I'esclavage des noirs qui conduit au massacre des blancs, la plupart des guerres maritimes et coloniales et même des guerres politiques, et de ce nombre : les sanglantes et inutiles luttes entre l'aristocratie anglaise et Napoléon ler, l'erreur de la campagne d'Égypte, celle de la conquête de l'Algérie, de

l'expédition du Mexique "(Jde, 1869, $15: 131)$.

Pourtant, les économistes favorables à la colonisation n'étaient qu'accessoirement soucieux de réfuter Malthus. D'une part, tant Batbié (Rddm, $1863: 883$ ) que Villiaumé (1867, II : 69) soulignèrent que I'essentiel n'était pas l'émigration, mais la colonisation et que le mouvement des capitaux devait accompagner celui des hommes. D'autre part, l'argument que l'émigration pouvait soulager la misère fut repris dans une tout autre perspective par Villiaumé en 1869 :

"M. Villiaumé dit en concluant que 6 millions de Français languissent dans l'indigence et que le moyen le plus prompt de les secourir serait de faire appel à ceux qui voudraient quitter la métropole, en leur offrant tous les moyens efficaces de fonder des établissements dans divers pays, mais surtout en Algérie, qui n'est point éloignée, et dont le climat est très salubre. Le système Wakefield pourrait être appliqué avec quelques modifications, selon la nature des pays à coloniser " (Jde, 1869, 15 : 133).

L'émigration était donc bien, avant tout, la condition nécessaire de l'expansion coloniale.

\section{Paix sociale ou émigration ?}

La question de la Paix sociale se raconte en deux actes. Acte premier, sous la Monarchie de Juillet l'opinion bourgeoise avait pris conscience de la misère ouvrière de l'industrie naissante, déjà observée en Angleterre depuis plusieurs décennies et l'on voyait les " effrayants progrès du paupérisme " atteindre la France. À cette misère étaient manifestement associées deux caractéristiques très bien documentées par les enquêtes sociales dont la plus retentissante fut celle de Louis René Villermé, qui publia en 1840 son Tableau de l'état physique et moral des ouvriers employés dans les manufactures de coton, de laine et de soie : une forte fécondité et une mortalité très élevée, surtout parmi les tout jeunes enfants, caractérisaient les ouvriers de " régime des manufactures " et les distinguaient de tous les autres groupes sociaux, de la bourgeoisie d'abord, mais aussi de l'immense masse des paysans, d'autant qu'en France, à l'inverse de l'Angleterre, le prolétariat rural était très limité. Les petites et moyennes propriétés, exploitées en faire-valoir direct, par fermage ou métayage étaient largement dominantes et l'on comptait en 1862 à peine 1400000 journaliers sur une population rurale de 26 millions d'âmes. Lorsqu'éclata en février 1848 la révolution qui renversa Louis-Philippe et instaura la Seconde République, les économistes montèrent en première ligne et utilisèrent sans relâche Malthus pour défendre l'ordre social : la cause de la misère des ouvriers, répétèrent-ils à l'envi, était leur trop grand nombre d'enfants, passant allègrement sous silence l'exceptionnelle gravité de la crise économique de 1846-1850, la chute vertigineuse des salaires et la brutale augmentation du chômage. 
Acte second, les élections de 1849, les premières en France au suffrage universel, qui révélèrent le conservatisme profond des paysans, soulagèrent la bourgeoisie, tandis que le bref épisode du Prince-Président Louis-Napoléon Bonaparte et le coup d'État de décembre 1851 confirmèrent le retour à l'ordre. $C^{\prime}$ en était fini du rêve socialiste. Aussitôt se mit en place une nouvelle ligne de défense idéologique. Les économistes commencèrent par mettre en cause le bienfondé des analyses démographiques différentielles : elles exagéraient le fossé entre les classes sociales. En témoignaient deux données jugées irréfutables : la "vie moyenne " s'était accrue en France et la fécondité de tous les autres groupes sociaux n'était nullement alarmante. Un argumentaire se construisit donc dès le début du Second Empire pour tirer le meilleur parti possible des données démographiques et en particulier de la faible lenteur de la croissance de la France. Si la société française ne connaissait pas les ravages du paupérisme, c'est parce que la Révolution de 1789 avait établi l'égalité entre tous et que le Code civil, grand œuvre napoléonien d'inspiration éminemment bourgeoise, avait instauré le partage successoral égalitaire, ce qui obligeait les paysans à restreindre leur fécondité pour éviter le morcellement de la propriété foncière à chaque succession. Une construction idéologique parfaitement cohérente s'en déduisait : la " sage " lenteur de la croissance démographique était l'écho de la " prudence " et du conservatisme des paysans français. La bourgeoisie y vit la preuve éclatante du bienfondé de la révolution de 1789 et du sacro-saint droit de propriété.

"La propriété territoriale [...] en multipliant le nombre des possesseurs du sol, [s'écria avec lyrisme en 1851 Moreau de Jonnès, le chef du bureau de la statistique] accroît le nombre des citoyens, défenseurs de la patrie et de l'ordre social qui s'élèvent au-dessus des prolétaires [...] et c'est là, bien plus que dans les cités, que gît la nation "

(Jde, 1851, $23: 321)$.

La paix sociale était à portée de main après la tempête de 1848, surtout avec un pouvoir autoritaire qui veillait sur les intérêts de la bourgeoise et donc, bien entendu, de la France.

Ce bref rappel historique est indispensable pour comprendre le prolongement international de la question de la paix sociale. La comparaison avec I'Angleterre prenait en effet une tout autre dimension. II était admis que l'émigration anglaise avait été rendue possible par le système d'héritage qui poussait les cadets à s'expatrier. Mais elle était aussi considérée comme le résultat de la pauvreté et dans le cas de I'Irlande, de la misère et la famine. Henri Baudrillart, professeur au Collège de France depuis 1852, ne manqua pas de relever que de telles conditions n'étaient guère réunies; bien au contraire :

" Pourquoi, par exemple nos 25 millions de paysans iraient-ils au loin chercher la terre à travers les difficultés et les risques de l'émigration et de la colonisation au prix d'une expatriation toujours pénible ? Cette terre ils la possèdent en toute sécurité, avec un revenu agricole qui depuis un demi-siècle est allé croissant ".

Par conséquent, déplorer la faiblesse de l'émigration française, c'était remettre en cause le droit de propriété, en raison de ses conséquences sur la fécondité : 
"Est-ce donc un mal, et faut-il au nom de l'émigration pousser la population à se développer sans mesure ? Au point de vue de la force et de la prospérité nationale, il est aujourd'hui bien connu que cette force et cette prospérité ne se mesurent pas au nombre de naissances, mais à la durée de la vie probable et moyenne. Les naissances qui aboutissent à des morts prématurées ne sont qu'une cause d'affaiblissement ; les enfants constituent non une richesse, mais une charge ".

Baudrillart en concluait logiquement que l'accroissement du niveau de vie était incompatible avec une politique coloniale aussi vigoureuse que celle de la Grande-Bretagne (Séances et travaux de I'Asmp, 1865 : 151-154). Ainsi le piège de la contradiction se refermait : les données socio-démographiques, qui fondaient la démonstration de la paix sociale à l'intérieur du pays, rendaient impossible une émigration indispensable à la conquête des marchés extérieurs et entravait les intérêts libre-échangistes. Dans ces conditions, il n'est pas étonnant que le thème de l'émigration soit resté relativement marginal. La contradiction se résoudra d'elle-même après 1870. D'abord, I'expansion coloniale aura pris une tout autre ampleur. Mais surtout, le déclin de la fécondité aura pris de telles proportions que les craintes pour la paix sociale fondées sur l'argument d'une excessive croissance démographique seront devenues tout à fait obsolètes. II en résultera l'abandon total du malthusianisme et le triomphe du populationnisme.

\section{La trop lente croissance démographique de la France}

À la fin du Second Empire, plusieurs économistes ont déjà évolué vers le populationnisme. Le cas de Baudrillart est le plus étonnant. Dans La liberté $d u$ travail, I'association et la démocratie, publié en 1865, il plaida éloquemment en faveur de l'émigration ${ }^{16}$. Les pays qui n'y avaient pas recours risquaient de connaître la décadence.

" Malheur aux nations qui se ferment aux rapports avec le reste du monde ! Malheur aux familles qui restreignent à l'excès le sein de leurs alliances! Elles trouvent dans l'abâtardissement physique et moral la confirmation, à leurs dépens, de cette loi providentielle qui fait de la fraternité humaine une vérité. En vain, l'humanité retenue au sol natal par mille attaches voudrait se soustraire à cette nécessité inévitable du mélange des idées et des races; il faut que des populations entières soient déracinées de leur patrie ".

Et il contrasta les souffrances endurées et les promesses du pays d'émigration. Cependant, l'argument décisif en faveur de l'immigration n'était pas humanitaire, mais économique : celle-ci profiterait à la France, en accroissant les profits. Il fallait donc que les Français émigrent :

"Pourquoi je le répète la France ne fournirait-elle pas l'émigration un plus vaste contingent? Est-il vrai que les Français soient si essentiellement sédentaires et casaniers qu'on l'a dit? De quel droit refuser à la nation française le titre de nation colonisatrice ?

Que l'on dise plutôt, en songeant à nos vieilles colonies et à l'Algérie, que c'est de l'administration et de l'excès de l'esprit réglementaire que sont venus nos échecs".

16 Les citations qui suivent sont tirées des pages 321-330. 
Nous n'avons donc plus affaire ici à une contradiction pure et simple, mais à un réel changement d'opinion. L'allusion à l'Algérie et à la responsabilité de l'administration ne sont pas fortuites. C'était la thèse des colons qui finira par l'emporter sur l'administration civile et militaire. Or, Baudrillart venait de découvrir le premier livre de Duval (1862) dont il louait les mérites et qui était précisément le représentant des intérêts coloniaux algériens. C'est donc au moins en partie sous son influence qu'il se déclara aussi favorable à l'émigration.

Louis Reybaud est intéressant d'un autre point de vue. Pour lui aussi l'émigration est le moyen et la colonisation, la fin :

"L'Amérique n'offre-t-elle pas aujourd'hui à l'Europe un déversoir naturel comme

I'Europe autrefois à l'Asie lorsque des fourmilières d'hommes débouchèrent des plateaux tartares ? L'humanité suit ainsi son double plan, qui est l'exploitation intégrale du sol et l'ennoblissement des espèces. D'un côté, les excédents de population vont animer les solitudes, de l'autre les types supérieurs remplacent les types inférieurs ; c'est sous l'empire de ces deux courants que le globe se peuple et se civilise "

(Rddm, 1866 : 986-987).

Mais s'il affirme que le ralentissement démographique de la France a rendu obsolète le point de vue de Malthus, il note pourtant que cette évolution n'est pas universelle : d'autres pays augmentent plus vite. D'où la conclusion, très nettement populationniste, que la population française s'accroît trop lentement :

"En France le problème n'est donc plus de mieux veiller sur soi-même ; peut-être y abonde-t-on déjà en excès, si l'on tient compte de l'équilibre à maintenir entre les influences territoriales... À tout prendre ce n'est pas la terre qui jusqu'ici a manqué aux hommes, ce sont plutôt les hommes qui ont manqué à la terre et sur ce point comme en tout il faut que leur mission s'accomplisse jusqu'au terme assigné ".

La sage croissance de la population française était devenue un grave handicap démographique.

\section{Une période privilégiée pour étudier l'émigration et la colonisation}

Jetons d'abord un rapide regard en arrière sur l'ensemble de la période 18401870. Pour la Monarchie de Juillet et la Seconde République (1840-1851), nous avons proposé une lecture du double combat idéologique mené par les économistes, à la fois contre l'aristocratie et la bourgeoisie protectionnistes, celles des industriels et des propriétaires terriens, et contre les socialistes. L'hostilité à l'encontre de l'émigration était fondée sur un argumentaire empirique et théorique centré sur la critique du "système protecteur ", bien trop étatiste à leurs yeux. L'État devait se cantonner à un strict rôle de gendarme et la libre circulation des hommes et des produits devait obéir aux seules lois du marché. Contre les socialistes, I'utilisation de l'argument démographique malthusien leur permit d'éluder la question de l'inégale répartition des richesses : trop d'ouvriers trop féconds recevaient nécessairement chacun une moindre part de la richesse. Quant à l'émigration et à la colonisation, dans cette construction l'État ne devait pas déroger à sa neutralité ni les individus espérer sortir du piège malthusien. 
Sous le Second Empire (1851-1870), se produit un profond revirement doctrinal et théorique qui reste parfaitement cohérent avec leurs fondamentaux idéologiques. Émigration et colonisation, devenues indissociables, apparurent comme un des moyens, voire une des conditions, de la croissance économique au moment de l'ouverture des frontières. Mais le contexte international révéla les limites des ambitions de libre-échangistes : avec la montée en puissance de la Prusse et surtout la prise de conscience de l'impérialisme anglais, la faible croissance démographique allait être un lourd handicap. Certains crurent qu'une colonisation " pacifique " serait la solution. La guerre de 1870 réduisit à néant ces illusions. Le plaidoyer pour l'émigration et la colonisation se heurtait à $d^{\prime}$ 'autres obstacles d'ordre idéologique. On ne pouvait en effet à la fois vanter les bienfaits de l'acquis majeur de 1789 , le droit de propriété source de la sage fécondité de la population française qui échappait ainsi au paupérisme et en même temps la déplorer en raison des enjeux politico-économiques de la colonisation. Or, le malthusianisme avait surtout son utilité dans le débat sur la paix sociale. En raison de l'impossibilité de réaliser une synthèse idéologiquement satisfaisante de ces contradictions, et comme le problème de la paix sociale ne fut plus après 1870 le moteur de l'évolution des idées sur la population, le déclin du malthusianisme était inéluctable. La fin du Second Empire est en réalité une période clef au cours de laquelle mûrissent de nombreuses composantes de I'idéologie bourgeoise de la Troisième République. Une ou deux décennies plus tard, lorsque la course coloniale deviendra intense et que la politique coloniale sera une affaire nationale, les idéologues échapperont à la contradiction qui consiste à attendre d'une faible croissance démographique qu'elle alimente simultanément le peuplement du pays et des colonies, en prônant une politique résolument nataliste et populationniste.

Finalement, la période étudiée se prête particulièrement à la mise en relation des faits et des idées, d'abord parce que les auteurs étudiés ici avaient à leur disposition un grand nombre de données factuelles, ce qui a ancré leur doctrine dans la réalité, contrairement par exemple aux socialistes utopiques, moins documentés en matière démographique. D'autre part, nous avons affaire à une véritable école de pensée, et non à des auteurs isolés, et dans un groupe aussi vivant, l'intégration des faits à la doctrine, tout comme les modifications doctrinales sous la pression des faits, est rapidement réalisée. Historiquement parlant, la période, on l'a vu, est à la fois riche et favorable aux élaborations tant théoriques que doctrinales. L'industrialisation, bien que modeste au regard des décennies ultérieures, bouleversait le champ du social aussi profondément que la révolution de 1789 avait transformé les cadres idéologiques et politiques. Quant au libre-échange, qui avait triomphé à partir de 1860 grâce à une série de traités bilatéraux, il fut définitivement aboli avec la loi Méline du 11 janvier 1892 : I'expérience libre-échangiste, imposée par le pouvoir impérial contre la volonté de la plus grande partie des milieux d'affaires, avait duré trente-deux ans. Les économistes, eux, avaient perdu leur raison d'être et il n'est pas surprenant qu'après 1870 le groupe s'affaiblisse et voit son influence diminuer. Des penseurs de premier plan tels qu'Émile Levasseur ou Paul Leroy-Beaulieu prennent quelque distance par rapport au groupe ; d'autres courants s'affirment, en particulier celui qu'anime la Société d'économie sociale et à partir de 1896 l'Alliance nationale pour l'accroissement de la population française où Bertillon s'impose. Enfin dès la fin des années 1890 Charles Gide et René Gonnard contestent, à travers la Revue d'économie politique, la prééminence du Journal des économistes. 
Par rapport aux décennies ultérieures, ces trente années se prêtent donc tout particulièrement à l'interprétation au niveau idéologique des relations entre faits et idées en matière d'émigration et de colonisation pour deux dernières raisons. D'une part la démographie au milieu du XIXe siècle, n'a pas encore acquis le statut de science autonome et relève à la fois de la théorie économique et de la doctrine sociale. Tout fait de population était donc naturellement interprété en termes économiques et sociaux. Les progrès ultérieurs de la statistique, au moment où l'idéologie populationniste s'affirmera bruyamment, accéléreront la dissociation entre théorie et doctrine démographiques. Comme d'autres sciences sociales, la démographie tendra à acquérir une certaine autonomie d'ordre "scientifique ". Certes, l'argument démographique, réputé scientifique, sera utilisé dans les débats de doctrine, mais cette extraordinaire limpidité du discours sur la population aura disparu. D'autre part, le milieu du XIXe siècle se caractérise par une rare transparence idéologique ce qui facilite considérablement la recherche. Nous I'avons vu à l'occasion de quelques citations, il suffit presque de donner la parole aux économistes. Le traumatisme de la Commune de 1870 et la montée des couches sociales chères à Léon Gambetta changeront la forme du discours social : celui-ci deviendra plus prudent donc plus opaque, et la bonne conscience triomphante de la bourgeoisie libérale se fera plus discrète, au moment où la critique marxiste dénoncera les leurres de l'idéologie dominante.

\section{Références bibliographiques}

\section{Sources primaires}

Ne figurent pas ici les articles publiés par les économistes dans diverses publications périodiques entre 1840 et 1870 . On les trouvera dans :

Annales de la Société d'économie politique (huit volumes, 1848-1870, publiés entre 1889 et 1895)

Journal de la Société de statistique de Paris (1860-1873)

La Revue des deux Mondes (1840-1870)

L'Économiste français (1860-1869)

Le Journal des économistes (1842-1872)

Les Mémoires de l'Académie des sciences morales et politiques (tomes 1 à 13, 1837-1872)

Les Séances et travaux de l'Académie des sciences morales et politiques (cinq séries, 1842-1872)

Batbié Anselme (1866) Nouveau Cours d'économie politique, Paris, Cotillon.

Baudrillart Henri (1872) Manuel d'économie politique, Paris, Guillaumin, 492 p.

Baudrillart Henri (1860) Des rapports de la morale et de l'économie politique, Paris, Guillaumin, $604 \mathrm{p}$. 
Bertillon Jacques (1885) La statistique humaine de la France, Paris, Baillière, $190 \mathrm{p}$.

Block Maurice (1869) L'Europe politique et sociale, Paris, Hachette, 628 p.

Boudin Jean-Christian-Marc-François-Joseph (1860) Éléments de statistique et de géographie générale, Paris, Plon, 232 p.

Boudin Jean-Christian-Marc-François-Joseph (1852) Statistiques de la population de la France et de ses colonies d'après les derniers recensements, Paris, Baillière, $56 \mathrm{p}$.

Dameth Henri (1869) Le mouvement socialiste et l'économie politique, Paris, Guillaumin, $142 \mathrm{p}$.

Duval Jules (1862) Histoire de l'émigration européenne asiatique et africaine au $X I X e$ siècle, Paris, Guillaumin.

Malthus Thomas Robert (1989 [1820]) Principles of Political Economy, edited by John Pullen, Cambridge, Cambridge University Press, 507 p.

Malthus Thomas Robert (1970 [1798]) An Essay on the Principle of Population, as it affects the future improvement of society, with remarks on the speculations of MR. Godwin, M. Condorcet and other writers, Harmondsworth, Penguin Books.

Malthus Thomas Robert (1965 [1826]) Essay on the Principle of Population, London, Dent, Everyman's Library.

Molinari Gustave de (1864) Cours d'économie politique, Paris, Guillaumin.

Molinari Gustave de (1854) Émigration, in Charles Coquelin et Gilbert Urbain Guillaumin Dirs., Dictionnaire de l'économie politique, Paris, Guillaumin, pp. 675-683.

Moreau de Jonnès Alexandre (1856) Éléments de statistique, Paris, Guillaumin, $464 \mathrm{p}$.

Passy Frédéric (1869) Les maux de la guerre et les bienfaits de la paix, Paris, Guillaumin, $108 \mathrm{p}$.

Passy Frédéric (s.d., en fait 1867) Conférence sur la paix et la guerre faite à l'école de médecine de Paris, Paris, Guillaumin.

Smith Adam (1970 [1776]) The Wealth of Nations, Harmondsworth, Penguin Books, $542 \mathrm{p}$.

Villiaumé Nicolas (1857) Nouveau traité d'économie politique, Paris, Guillaumin.

\section{Sources secondaires}

Armengaud André (1962) L'opinion publique en France et la crise nationale allemande en 1866, Paris, Les Belles Lettres, 119 p.

Bairoch Paul (1976) Commerce extérieur et développement économique de I'Europe, Paris-La Haye, Mouton, 355 p.

Breton Alain et Lutfalla Michel (Dirs.) (1991) L'économie politique en France au $X I X e$ siècle, Paris, Economica, 680 p.

Brunschwig Henri (1949) La colonisation française, du pacte colonial à I'Union française, Paris, Calmann-Lévy. 
Charbit Yves (2014) Le passé français : protectionnisme et concurrence (XVe$\mathrm{XIXe)}$, in Martine Behar-Touchais, Nicolas Charbit et Rafael Amaro Éds., À quoi sert la concurrence? Paris, Institut du droit de la concurrence, pp. 111-116.

Charbit Yves (2010) The Classical Foundations of Population Thought from Plato to Marx, Dordrecht, Springer, $179 \mathrm{p}$.

Charbit Yves (2001) Migration et colonisation dans la pensée de Platon, Revue Européenne des Migrations Internationales, 17 (3), pp. 199-210.

Charbit Yves (1981) Du malthusianisme au populationnisme. Les Économistes français et la population 1840-1870, Paris, INED/PUF, 307 p.

Duroselle Jean-Baptiste (1951) Les débuts du catholicisme social en France (1822-1870), Paris, PUF, 787 p.

Fohlen Claude (1956) L'industrie textile au temps du Second Empire, Paris, Plon, $534 \mathrm{p}$.

Galbraith John S. (1970) Myths of "Little England" Era, in Alan Shaw and George Lewers Eds., Great-Britain and the Colonies, London, Methuen, pp. 27-45.

Gosh Rabindra N. (1964) The Colonisation Controversy: R.J. Wilmot-Horton and the Classical Economists, Economica, 31 (124), pp. 385-400.

Hobsbawm Eric (1977) The Age of Revolution. Europe 1789-1848, London, Abacus Press, 216 p.

Julien Charles-André (1964) Histoire de l'Algérie contemporaine, Paris, PUF, $631 \mathrm{p}$.

Kreager Philip (2008) Aristotle and Open Population Thinking, Population and Development Review, 34 (4), pp. 599-629.

Levasseur Pierre Émile (1859) Histoire des classes ouvrières en France depuis la conquête de Jules César jusqu'à la révolution, tome premier, Paris, Guillaumin, $587 \mathrm{p}$.

Shaw Alan George Lewers (1970) Great-Britain and the Colonies, London, Methuen.

Spengler Joseph J. (1936) French population theory since 1800, The Journal of Political Economy, October, pp. 577-611 ; December, pp. 743-766.

Winch Donald N. (1963) Classical Economy and the Case for Colonisation, Economica, 30 (120), pp. 387-399. 


\section{Yves Charbit}

\section{Émigration, colonisation et idéologie libérale en France (1840-1870)}

Au milieu du XIXe siècle (1840-1870), les théories et les doctrines relatives à l'émigration sont indissociables de la question de la colonisation, en particulier dans les écrits des économistes libre-échangistes français, les meilleurs spécialistes de leur temps. L'article montre que pour analyser l'évolution des idées, il est indispensable de s'attacher au contexte historique et à deux faits majeurs, la conquête de l'Algérie et la rivalité impérialiste avec l'Angleterre. Sur le plan doctrinal, il en est résulté l'abandon du malthusianisme, jusqu'alors dominant, puisque la faible croissance démographique française ne présentait plus comme avant 1848 un danger pour la paix sociale et que le problème était au contraire l'insuffisance prévisible des ressources humaines nécessaires pour peupler l'empire colonial en plein essor.

\section{Emigration, Colonization and Liberal Ideology in France (1840-1870)}

In the middle of the 19th century (1840-1870), theories and doctrines on migration were intertwined with those on colonization, as is particularly clear in the writings of the French free-traders, the best specialists of their time. This paper shows that in order to analyze the evolution of their ideas, it is a crucial to take into account the historical context and notably the conquest of Algeria and the imperial rivalry with England. It follows that Malthusianism, up to then the dominant doctrine, was abandoned because the slow demographic growth was no longer a threat to social peace as was the case before 1848 while human resources were likely to be insufficient to people the rapidly expanding colonial empire.

\section{$\cdots$ Emigración, colonización e ideología liberal en Francia (1840-1870)}

A mediados del siglo XIX (1840-1870), las teorías y doctrinas relativas a la emigración son indisociables de la cuestión de la colonización, en particular en los escritos de los economistas franceses partidarios del libre intercambio que eran los mejores especialistas de la época. El artículo muestra que para analizar la evolución de las ideas es indispensable interesarse al contexto histórico y a dos eventos de gran importancia: la conquista de Argelia y la rivalidad imperialista con Inglaterra. En el plano doctrinal, cabe destacar el abandono del malthusianismo, hasta entonces dominante, dado que el débil crecimiento demográfico en Francia ya no presentaba mayores riesgos para la paz social, como sí era el caso antes de 1848. Por el contrario, el problema era la previsible insuficiencia de los recursos humanos disponibles para poblar el imperio colonial en plena expansión. 